\title{
START CONDITION AND NON-LINEAR SATURATION IN HIGH CURRENT FEL-OSCILLATOR
}

\author{
V.K.Grishin", T.A.Novicova \\ Institute of Nuclear Physics of Moscow Lomonosov State University
}

\begin{abstract}
Properties of high current free electron laser (FEL) with a collective regime of electromagnetic wave excitation are considered. Start value of electron current in a FELoscillator and non-linear saturation of field excitation are estimated. Possibility of such device creation on base MSU electron linear accelerator is discussed.
\end{abstract}

\section{INTRODUCTION}

In present, several high current FEL facilities are under construction and operation ( see for example [1], in particular [2,3]). A high current FEL has a high coefficient of signal amplification, and this permits to extend the possibilities to create the coherent short-wave radiation sources. The high signal amplification is a result of a collective interaction of an electron beam with an electromagnetic wave. The latter can be realised if the density of electron is sufficiency high. With that an intense beam acquires the properties of optical waveguide, and the electromagnetic radiation is concentrated in the beam volume [4]. Therefore as it has been shown in previous paper [5], a high current regime can be realised in FEL having a moderate current but low emittances of particles that is peculiar for an electron accelerators of new generation.

In present paper the high current approach is being used to analyse the peculiarity of a scheme of FEL-Oscillator and to estimate its start and saturation conditions. It is of interest can we have here any application of this scheme on the MSU electron accelerator?

\section{START CONDITION IN HIGH CURRENT FEL OSCILLATOR}

Consider a traditional scheme of a charged beam generator. A system constructed along the axis $\mathbf{z}$ consists of undulator with a period $\lambda_{0}$ and length $L_{\text {und }}$ which is located between two mirrors. The mirrors separated by distance $\mathbf{L}_{\mathbf{m}}\left(\mathbf{L}_{\mathbf{m}}>\mathbf{L}_{\mathbf{u n d}}\right)$ are forming an open resonator . Along the axis $\mathbf{z}$ in a point placed before the undulator a continuous beam of monoenergetic electrons is being

\footnotetext{
*Email: grishin@depni.npi.msu.su
}

injected into the system (by means of supplementary magnet system). The electron energy is $E=m c^{2} \gamma_{L}$ where $\gamma_{\mathrm{L}}$ is Lorenz's factor, the initial electron velocity is $\mathrm{v}_{0}=\beta_{0} \mathrm{c}$. Passing trough the undulator the electrons acquire a transversal velocity $v_{\perp}=\beta_{\perp} \mathrm{c}$ that permits them to interact with an optical (i.e. transversal) wave. After passage he undulator the electron beam is being deflected from the system (by means of second supplementary magnets).

Analyse start condition of high current FEL oscillator. Let an initial signal of frequency $\omega$ be brought in the system. The signal passing through the undulator can be amplified by an electron beam and then being partly irradiated and reflected from the mirrors returns to the initial point. Usually a start condition of FEL oscillator is estimated from one if the amplification of circling signal begins surpass its total absorption. But the high current FEL has its important peculiarities.

An electron beam in the undulator is an electromagnetic medium in which some transversal waves of different modes can propagate in both directions. An initial signal is distributed among the co-travelling with beam waves some of which can interact with the electrons. Its interaction becomes resonant if the double conditions are carried out:

$$
\omega=\mathrm{kc} ; \mathrm{k}=\mathrm{k}_{0} \beta_{0 \mathrm{Z}} /\left(1-\beta_{0 \mathrm{Z}}\right)
$$

where $\mathbf{k}$ is a wave vector, $\mathrm{k}_{0}=2 \pi / \lambda_{0}$, $\beta_{0 Z}=\left(\beta_{0}^{2}-\beta_{\perp}^{2}\right)^{1 / 2}$.

Practically one can consider only the mostly amplified wave among the co-travelling ones after passing the undulator and only one back travelling wave because an electron beam do not disturb the electromagnetic oscillations in last case.

Hence the final balance of amplification and absorption in system gives the nest condition of reliable generation start (see [6] too):

$$
\mathrm{R}_{1} \mathrm{R}_{2} \exp \left(\mathrm{i} \int_{0}^{\mathrm{L}_{\mathrm{m}}}\left(\mathrm{k}^{+}-\mathrm{k}^{-}\right) \mathrm{dz}\right)=1
$$

where $R_{1,2}$ are the coefficients of wave amplitudes reflection from the first and second mirrors respectively (value of $R_{2}$ includes the loss of signal due to its 
irradiation ), $\mathrm{k}^{+}$and $\mathrm{k}^{-}$are the wave vectors of forward and back directions for given frequency and mode. Here we take into account that the waves coming in and out from an electron beam are almost not reflected. Outside electron beam

$$
\mathrm{k}^{+}=\mathrm{k}=\frac{\omega}{\mathrm{c}}, \mathrm{k}^{-}=-\frac{\omega}{\mathrm{c}}
$$

and inside one

$$
\mathrm{k}^{+}=\mathrm{k}+\delta \mathrm{k}^{+} ; \quad\left|\delta \mathrm{k}^{+}\right|<<\mathrm{k}
$$

Hence the equation (2) is reduced to

$$
2 \mathrm{~kL}_{\mathrm{m}}+\delta \mathrm{k}^{+} \mathrm{L}_{\text {und }}=2 \pi \mathrm{M}+\mathrm{i} \ln \left|\mathrm{R}_{1} \mathrm{R}_{2}\right|
$$

where $\mathrm{M}=\mathrm{S}-\arg \left(\mathrm{R}_{1} \mathrm{R}_{2}\right) / 2 \pi, \quad \mathrm{S}$ is an integer. Separating the real and imaginary parts of (4) one can write

$$
\begin{aligned}
& 2 \mathrm{~kL}_{\mathrm{m}}+\operatorname{Re}\left(\delta \mathrm{k}^{+}\right) \mathrm{L}_{\text {und }}=2 \pi \mathrm{M} \\
& \operatorname{Im}\left(\delta \mathrm{k}^{+}\right) \mathrm{L}_{\text {und }}=-\ln \left|\mathrm{R}_{1} \mathrm{R}_{2}\right|
\end{aligned}
$$

This equation is written for a given value of frequency. And the first equation in (6) determines conditions when the wave excitation of given mode is possible in beam system. But already after the first wave circulation, the excitation of electromagnetic oscillations in mirror resonator goes to play the important role. Therefore it is necessary to add the condition of wave excitation in FEL resonator with the same value of frequency. Such condition produces a relation [7]

$$
2 \mathrm{~kL}_{\mathrm{m}}=2 \pi(\mathrm{N}+(\mathrm{n}+\mathrm{q}+1) / 2+\Delta)
$$

where $\mathrm{N}, \mathrm{n}, \mathrm{q}$ are the integers, $\Delta$ is a mode shift determined by resonator parameters.

The second equation in (6) determines the start condition for the electron beam current $I_{b}$. Really according with a solution of beam linear system the increment of wave excitation $\operatorname{Im}\left(\delta \mathrm{k}^{+}\right)=-\mathrm{AI}_{\mathrm{b}}^{\alpha}$ ( see below). Due to this the second condition in (6) drives to the direct dependence of start value of $I_{b}$ on $R_{1,2}, A$, and $\alpha$ ones:

$$
\mathrm{I}_{\mathrm{b}_{\mathrm{st}}}=\left(\frac{\ln \left|\mathrm{R}_{1} \mathrm{R}_{2}\right|^{-1}}{\mathrm{AL}_{\text {und }}}\right)^{1 / \alpha}
$$

The magnitude of $\alpha \approx 1 / 3-2 / 5$ for a wide and compressed beam respectively [5]. For the latter case

$$
A=k_{0}\left(\frac{4}{\mathrm{ka}_{\mathrm{b}} \mathrm{I}_{\mathrm{A}}}\right)^{\alpha}
$$

where $\mathrm{I}_{\mathrm{A}}=17 \mathrm{kA}$, and $\mathrm{k}=\mathrm{k}_{0} \gamma_{0}^{2}$.

The value of $R_{2}=(1-\varepsilon)^{1 / 2}$ where factor $\varepsilon<<1$ including the fraction of electromagnetic flow irradiated through the second mirror. To determine the value of $R_{1}$ it is necessary to take into analysis the fact of strong channelling of electromagnetic radiation in the beam volume. As a result the cross-section of electromagnetic flow becomes comparable with of beam one. The electromagnetic flow reflected from the second mirror returns to the first one and then to the undulator expanding due to a diffraction. As a result the fraction of electromagnetic flow returned in the beam volume is reduced to [8]

$$
\mathrm{F}=\left(\frac{\mathrm{a}_{\mathrm{b}} \mathrm{a}_{\mathrm{E}} \mathrm{k}}{2 \mathrm{~L}_{\mathrm{d}}}\right)^{2}
$$

where $a_{b, E}$ are the radius of beam and electromagnetic flow canalised in it, $\mathrm{L}_{\mathrm{d}}=2 \mathrm{~L}_{\mathrm{m}}-\mathrm{L}_{\text {und }}$.

Finally

$$
\mathrm{R}_{1}=\mathrm{F}^{1 / 2} / \mathrm{N}^{+}
$$

where $\mathrm{N}^{+}$is the number of forward travelling waves in beam system (usually $\mathrm{N}^{+}=2-3[5,6]$ ).

On the whole the collective regime indicates in FEL a relatively weak dependence of start current value on the resonator parameters.

Basing on the formulas obtained above one can get a simple estimate of increment value needed for lasing. It is not difficult to see that the spatial distribution of radiation field outside of electron beam is been described by function

$$
\mathrm{E}\left(\mathrm{r}>\mathrm{a}_{\mathrm{b}}\right) \approx \mathrm{K}_{0}(\mathrm{vr})
$$

where $K_{0}$ is Bessel function of imaginary value, $v^{2}=\mathrm{k}^{2}$ $(\omega / c)^{2}$. Therefore the radiation beam radius $a_{E} \approx 1 / v$. Using a relation $\operatorname{Im}\left(\delta \mathrm{k}^{+}\right) \approx v^{2} /(2 \mathrm{k})$ we see that $\operatorname{Im}\left(\delta \mathrm{k}^{+}\right) \approx$ $1 /\left(2 \mathrm{ka}_{\mathrm{E}}{ }^{2}\right)$. In result the quantity

$$
\zeta=\operatorname{Im}\left(\delta \mathrm{k}^{+}\right) \mathrm{L}_{\text {und }}
$$

is determined by the equation

$$
\zeta \approx \ln \zeta+\ln \sigma / 2+\eta
$$

where $\sigma=\mathrm{a}_{\mathrm{E}} / \mathrm{a}_{\mathrm{b}}$, a parameter $\eta \approx 1,5-3$. Thus the start value of $\zeta_{s t} \geq 2-3$.

\section{SOME COMMENTARIES. NON-LINEAR SATURATION}

Notice some important effects of collective FEL. Resonance electron accelerators have a micropeaks longitudinal structure of beam with frequency of peaks repetition at several GHz. But its length exceeds the excited electromagnetic wave ones very considerable. Therefore all mentioned estimates retain correct. But the 
main practical problem is an exact concordance the return of electromagnetic signal with the injection of next electron peaks. Besides the necessity of fine tuning of optical resonator it is important to take into account that, due to an intense beam acquires in undulator the properties of optical waveguide, the phase velocity $\mathrm{v}_{\mathrm{ph}}$ of excited electromagnetic wave is been reduced, and

$$
\mathrm{v}_{\mathrm{ph}}=\mathrm{c}\left(1-\operatorname{Re} \delta \mathrm{k}^{+} / \mathrm{k}\right)
$$

It is of interest that this circumstance is very useful for to avoid a problem of lethargy or "anti-lethargy" [9].

Then discuss one important non-linear effect. How long is continuing the process of electromagnetic energy accumulation in FEL resonator? Taking into account the mechanism of electron-electromagnetic field interaction one can conclude that a process of electron energy transfer to a radiation goes to be saturated after electron trapping by the ponderomotive wave. In spite of resonant tuning, the velocity of ponderomotive wave becomes less (according with (15)) that the beam one. Longitudinal braking of particles is been caused by the interaction of particle with magnetic field $\mathrm{H}$ of exited electromagnetic wave and is been described by relation

$$
m c^{2} \gamma \gamma_{z}^{2} \frac{d \beta_{z}}{d t}=-\mathrm{ev}_{\perp} \mathrm{H}
$$

Therefore trapping of electrons can realise if the mean electric field $\langle\mathrm{E}\rangle$ reaches to the next saturation level (see (9)):

$$
<\mathrm{E}>\approx \frac{\mathrm{AI}_{\mathrm{b}}^{\alpha} \mathrm{I}_{\mathrm{A}} \gamma^{5} \beta_{\mathrm{z}}}{2 \mathrm{ck}_{0} \mathrm{~L}_{\text {und }}}
$$

Dependence of $\langle E\rangle$ value on FEL parameter is evidence.

\section{FEL OSCILLATOR ON BASE OF MSU ELECTRON ACCELERATOR}

In present time in Institute of Nuclear Physics of MSU there is a continuous wave electron accelerator with the particle energy of 1.2-2.4 $\mathrm{MeV}$. The device is designed for different atomic and nuclear and applied researches. It produces a continuous electron beam with the average current magnitude up to 10-30 $\mathrm{mA}$ and small emittance.. The beam has a peak microstructure (frequency of repetition is $2.45 \mathrm{GHz}$ ) with peak current magnitude up to 0.4-1.5 A.

The values of $\gamma_{0}=3.4-5.6$ permit to use this accelerator for producing the electromagnetic radiation in millimeter and submillimeter ranges what opens a very important applications in biology and so on. Estimate the start current value.
Let the system and beam parameters be the next: $\lambda_{0}=2$ $\mathrm{cm}, \mathrm{L}_{\mathrm{m}}=2 \mathrm{~L}_{\text {und, }} \mathrm{a}_{\mathrm{b}}=0.1 \mathrm{~cm}, \mathrm{a}_{\mathrm{E}}=2-3 \mathrm{a}_{\mathrm{b}}$. Then for a real continuous beam the value of $\mathrm{I}_{\mathrm{bst}} \cong 30 \mathrm{~mA}$ is been reached with $\mathrm{L}_{\text {und }} \cong 200 \mathrm{~cm}$ and $250 \mathrm{~cm}$ for the electron energies 1.2 and 2.4 MeV respectively. Thus this accelerator can be used as FEL oscillator.

\section{REFERENCES}

[1] Proceedings of the 1995 Particle Accelerator Conference, IEEEAPS, Synchrotron Light Sources and FREE Electron Lasers, v.1 , pp.155-311,

[2] M.Hogan, C,Pellegrini, J. Rjsenzweig et al., ibid. p. 240.

[3] D. Baunan<, D. Jianjiun, H. Shenzong et al., ibid, p.246.

14] J.M.J.Madey, J.App. Phys., v.42 (1971),1906.

[5] V.K.Grishin, B.S.Ishkhanov. T.A.Novicova, V.I.Shvedunov, idid, p. 225.

[6] E.M.Lifshits, L.P.Pitaevski, Physical Kinetics, $\$ 65$, Nauka Press, Moskva.

[7] O.Zvelto, Principles of Lasers, Plenum Press, New York.

[8] L.D.Landau, E.M.Lifshits, Theory of Field, § 61, Nauka Press, Moskva.

[9] T.C.Marshall, Free-Electron Lasers, New York, 1985. 